\title{
PROPERTIES OF THE MODULUS OF CONTINUITY FOR MONOTONOUS CONVEX FUNCTIONS AND APPLICATIONS
}

\author{
SORIN GHEORGHE GAL \\ Department of Mathematics \\ University of Oradea \\ Str. Armatei Romane 5 \\ 3700 Oradea, Romania
}

(Received January 29, 1992 and in revised form November 22, 1992)

\begin{abstract}
For a monotone convex function $f \in C[a, b]$ we prove that the modulus of continuity $\omega(f ; h)$ is concave on $[a, b]$ as function of $h$. Applications to approximation theory are obtained.
\end{abstract}

KEY WORDS AND PHRASES. Concave modulus of continuity, approximation by positive linear operators, Jackson estimate in Korneichuk's form.

1991 AMS SUBJECT CLASSIFICATION CODES. 41A10, 41A36, 41A17.

\section{INTRODUCTION.}

In a recent paper, Gal [1] the modulus of continuity for convex functions is exactly calculated, in the following way.

THEOREM 1. (see [1]) Let $f \in C[a, b]$ be monotone and convex on $[a, b]$. For any $h \in[0, b-a]$ we have:

(i) $\omega(f ; h)=f(b)-f(b-h)$, if $f$ is increasing on $[a, b]$,

(ii) $\omega(f ; h)=f(a)-f(a+h)$, if $f$ is decreasing on $[a, b]$,

where $\omega(f ; h)$ denotes the classical modulus of continuity.

Denote

$K M[a, b]=\{f \in C[a, b] ; f$ is monotonous convex or monotonous concave on $[a, b]\}$

The purpose of the present paper is to prove that for $f \in K M[a, b]$ the modulus of continuity $\omega(f ; h)$ is concave as function of $h \in[0, b-a]$ and to apply this result to approximation by positive linear operators and to Jackson estimates in Korneichuk's form.

\section{MAIN RESULTS AND APPLICATIONS.}

A first main result is the following

THEOREM 2. For all $f \in K M[a, b]$, the modulus of continuity $\omega(f ; h)$ is concave as function of $h \in[0, b-a]$.

PROOF. Let firstly suppose that $f$ is increasing and convex on $[a, b]$. If $f$ is increasing on $[a, b]$, by Theorem $1,(\mathrm{i})$, we have $\omega(f ; h)=f(b)-f(b-h)$. Hence

and

$$
\begin{gathered}
\alpha \omega\left(f ; h_{1}\right)+(1-\alpha) \omega\left(f ; h_{2}\right)=f(b)-\alpha f\left(b-h_{1}\right)-(1-\alpha) f\left(b-h_{2}\right) \\
\omega\left(f ; \alpha h_{1}+(1-\alpha) h_{2}\right)=f(b)-f\left(b-\alpha h_{1}-(1-\alpha) h_{2}\right)
\end{gathered}
$$

for all $\alpha \in[0,1]$ and all $h_{1}, h_{2} \in[0, b-a]$. 
Since $f$ is convex on $[a, b]$ we get

$$
f\left(b-a h_{1}-(1-\alpha) h_{2}\right) \leq \alpha f\left(b-h_{1}\right)+(1-\alpha) f\left(b-h_{2}\right) .
$$

wherefrom taking into account (1.1) and (1.2) too, we get

$$
\alpha \omega\left(f ; h_{1}\right)+(1-\alpha) \omega\left(f ; h_{2}\right) \leq \omega\left(f ; \alpha h_{1}+(1-a) h_{2}\right)
$$

Now, if $f$ is decreasing on $[a, b]$, since by Theorem 1 , (ii), we have $\omega(f ; h)=f(a)-f(a+h)$, we immediaiely get

and

$$
\alpha \omega\left(f ; h_{1}\right)+(1-\alpha) \omega\left(f ; h_{2}\right)=f(a)-\alpha f\left(a+h_{1}\right)-(1-\alpha) f\left(a+h_{2}\right)
$$

$$
\omega\left(f ; \alpha h_{1}+(1-\alpha) h_{2}\right)=f(a)-f\left(a+\alpha h_{1}+(1-\alpha) h_{2}\right)
$$

for all $\alpha \in[0,1]$ and all $h_{1}, h_{2} \in[0, b-a]$.

Since $f$ is convex on $[a, b]$ we have

$$
f\left(a+\alpha h_{1}+(1-\alpha) h_{2}\right) \leq \alpha f\left(a+h_{1}\right)+(1-\alpha) f\left(a+h_{2}\right),
$$

which together with (1.4) and (1.5) gives again (1.3).

In the following we need the

DEFINITION 1. (see e.g. [2]) Let $f \in C[a, b]$ be. If $\omega(f ; h)=\sup \{|f(x)-f(y)| ;|x-y| \leq h\}$ is the usual modulus of continuity, the least concave majorant of $\omega(f ; h)$ is given by

$$
\widetilde{\omega}(f ; h)=\sup \left\{\frac{(\delta-\alpha) \omega(f ; \beta)+(\beta-\delta) \omega(f ; \alpha)}{\beta-\alpha} ; 0 \leq \alpha \leq \delta \leq \beta \leq b-a\right\} .
$$

An immediate consequence of Definition 1 is the

COROLLARY 1 . For any $f \in K M[a, b]$ we have

$$
\widetilde{\omega}(f ; h)=\omega(f ; h)
$$

PROOF. Putting $\alpha=\delta$ in Definition 1 we get

$$
\omega(f ; h) \leq \widetilde{\omega}(f ; h) .
$$

Then, taking into account Theorem 2, for $0 \leq \alpha \leq \delta \leq \beta \leq b-a$ we have

$$
\frac{(\delta-\alpha) \omega(f ; \beta)+(b-\delta) \omega(f ; \alpha)}{\beta-\alpha} \leq \omega\left(f ; \frac{\beta(\delta-\alpha)}{\beta-\alpha}+\frac{\alpha(\beta-\delta)}{\beta-\alpha}\right)=\omega(f ; \delta)
$$

wherefrom passing to supremum, we immediately get

$$
\widetilde{\omega}(f ; \delta) \leq \omega(f ; \delta),
$$

which proves the corollary.

REMARK. It is easy to see that Corollary 1 remains valid for all $f \in C[a, b]$ having a concave modulus of continuity $\omega(f ; h)$.

Now, firstly we will apply the previous results to approximation by positive linear operators.

Thus, investigating the sequence of Lehnhoff polynomials in [3], $L_{n}(f)(x)$, defined for $f \in C[-1,1]$, H.H. Gonska [2] proves that

$$
\left|L_{n}(f)(x)-f(x)\right| \leq \sqrt{\frac{30}{11}} \widetilde{\omega}\left(f ; \frac{\sqrt{1-x^{2}}}{n}+\frac{|x|}{n^{2}}\right)
$$

Taking now into account Corollary 1 we immediately get the 
COROLLARY 2. If $f \in K M[-1,1]$ then for all $x \in[-1,1], n \in N$ we have

$$
\left|L_{n}(f)(x)-f(x)\right| \leq \sqrt{\frac{30}{11}} \omega\left(f: \frac{\sqrt{1-x^{2}}}{n}+\frac{|x|}{n^{2}}\right)
$$

In the same paper, for $f \in C[0,1]$, H.H. Gonska obtains estimates in terms of the modulus $\widetilde{\omega}(f ; h)$ in the approximation by the so-called Shepard operator, $S_{n}^{\mu}(f), 1 \leq \mu \leq 2$. Then by Corollary 1 and by Theorem 4.3 in [2] we immediately get the

COROLLARY 3. For all $f \in K M[0,1]$ and all $n \in N$ we have

$$
\begin{gathered}
\left\|S_{n}^{1}(f)-f\right\| \leq \frac{n+1}{n} \omega\left(f ; \frac{1}{\ln (2 n+2)}\right) \\
\left\|S_{n}^{\mu}(f)-f\right\| \leq \frac{14}{2-\mu} \omega\left(f ; \frac{1}{(n+1)^{\mu-1}}\right), 1<\mu<2 \\
\left\|S_{n}^{2}(f)-f\right\| \leq 19 \omega\left(f ; \frac{\ln (n+1)}{n+1}\right) .
\end{gathered}
$$

Finally, we will apply our results to the following so-called Jackson estimate in Korneichuk's form.

THEOREM 3. (see e.g. [4], p. 147) For any $f \in C[-1,1]$ we have

$$
E_{n-1}(f) \leq \omega\left(f ; \frac{\pi}{n}\right), n=1,2, \cdots,
$$

where $E_{k}(f)$ denotes the best approximation by polynomials of degree $\leq k$.

Now, we will prove the

THEOREM 4. If $f \in C[-1,1]$ has a concave modulus of continuity $\omega(f ; h), h \in[0,2]$, then we have

$$
E_{n-1}(f) \leq \frac{1}{2} \omega\left(f ; \frac{\pi}{n}\right)
$$

PROOF. Extending $\omega$ to $[0, \pi]$ by taking $\omega(f ; h)=\omega(f ; 2), h \in[2, \pi]$, obviously $\omega$ remains concave on $[0, \pi]$.

Denote $\omega(h)=\omega(f ; h), h \in[0, \pi]$ and

$$
\Lambda_{\omega}=\{g \in C[-1,1] ; \omega(g ; h) \leq \omega(h), \forall h \in[0, \pi]\} .
$$

Obviously $f \in \Lambda_{\omega}$. Then by [5, Theorem 8 and Lemma 2, p. 122-123], as in the proof of Theorem 9 , p. 123 in [5], there is $g \in \operatorname{Lip}_{M^{1}}$ such that

$$
\|f-g\| \leq \frac{1}{2} \omega\left(f ; \frac{\pi}{n}\right)-\frac{\pi M}{2 n} .
$$

Now by Theorem V, (ii), in [4, p. 147], there is $P_{n-1}$ polynomial of degree $\leq n-1$ such that

$$
\left\|g-P_{n-1}\right\| \leq \frac{\pi M}{2 n} .
$$

Hence we get

$$
\left\|f-P_{n-1}\right\| \leq\|f-g\|+\left\|g-P_{n-1}\right\| \leq \frac{1}{2} \omega\left(f ; \frac{\pi}{n}\right)
$$

which proves the theorem.

REMARK. For $f \in K M[-1,1]$, Theorem 4 remains valid. 


\section{REFERENCES}

1. GAL, S.G., Calculus of the modulus of continuity for nonconcave functions and applications, Calcolo 27 (1990), 195-202.

2. GONSKA, H.H., On approximation in spaces of continuous functions, Bull. Austral. Math. Soc. 28 (1983), 411-432.

3. LEHNHOFF, H.G., A simple proof of A.F. Timan's theorem, J. Approx. Theory 38 (1983), $172-176$.

4. CHENEY, E.W., Introduction to Approximation Theory, McGraw-Hill Book Company, New York, 1966.

5. LORENTZ, G.G., Approximation of Functions, Holt, Rinehart and Winston, Second Edition, New York, 1986. 


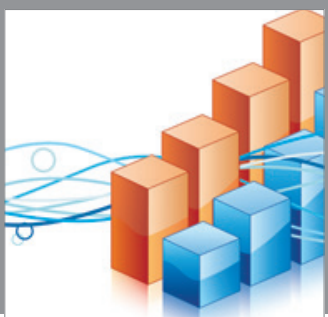

Advances in

Operations Research

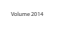

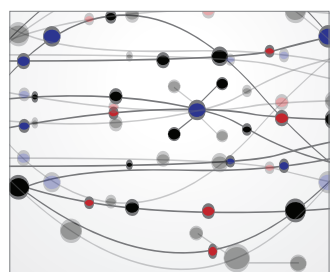

\section{The Scientific} World Journal
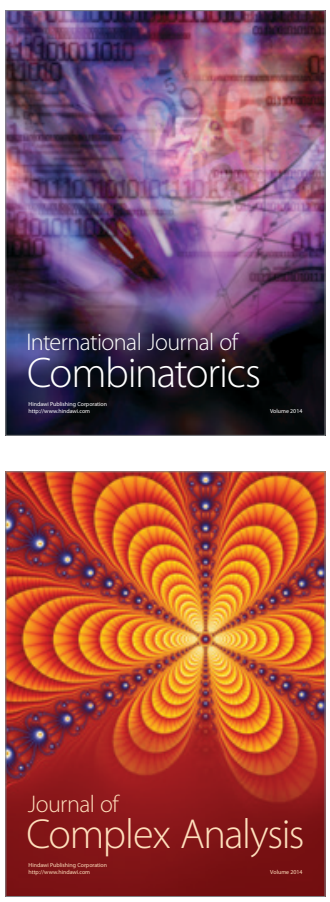

International Journal of

Mathematics and

Mathematical

Sciences
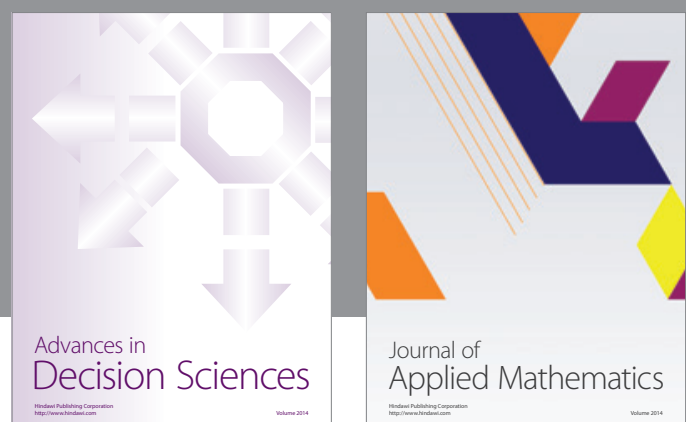

Journal of

Applied Mathematics
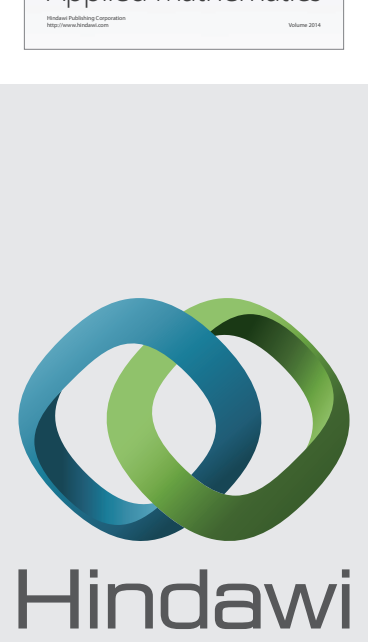

Submit your manuscripts at http://www.hindawi.com
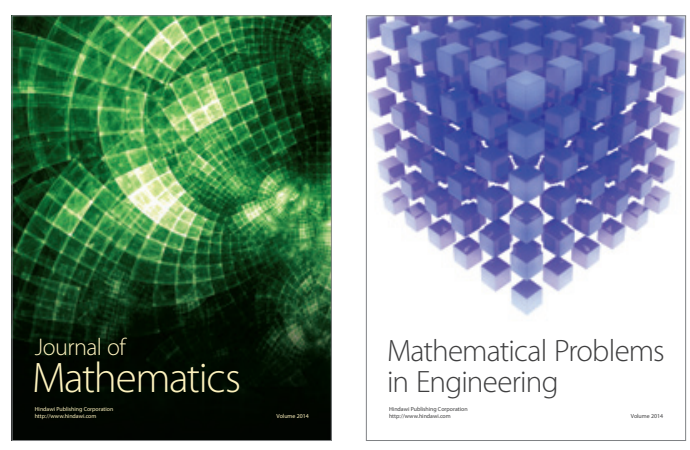

Mathematical Problems in Engineering
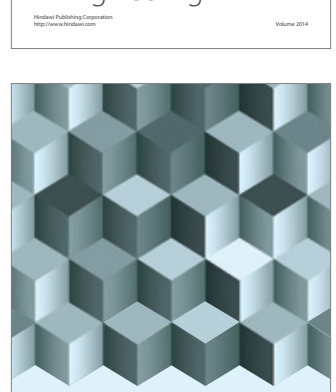

Journal of

Function Spaces
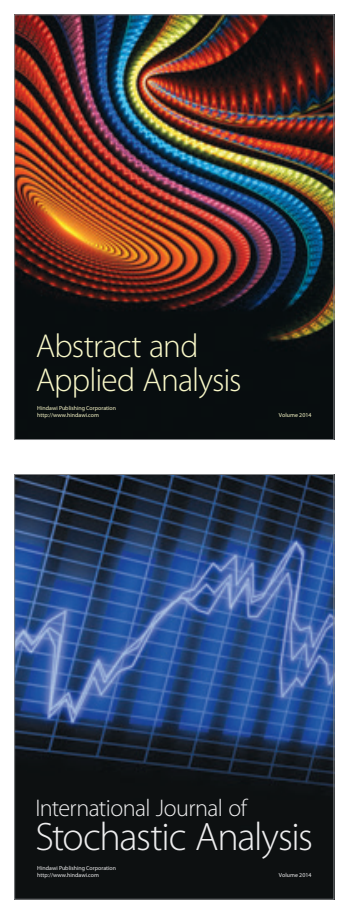

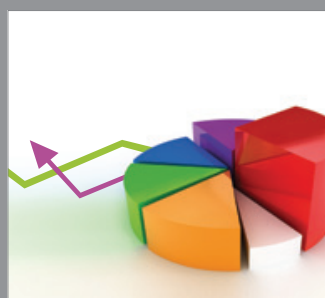

ournal of

Probability and Statistics

Promensencen
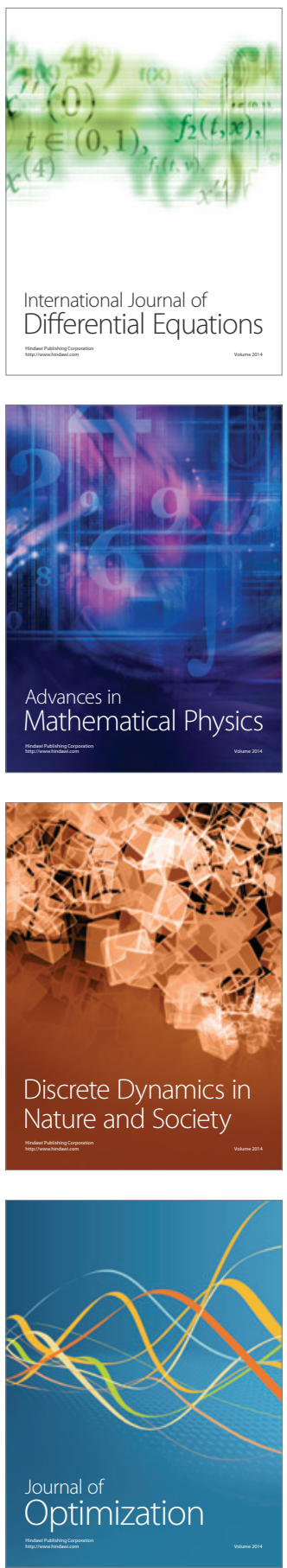